\title{
Target analysis and retrospective screening of mycotoxins and pharmacologically active substances in milk using an ultra-high-performance liquid chromatographyl high-resolution mass spectrometry approach
}

\author{
Luana Izzo, ${ }^{1}$ ๑ Yelko Rodríguez-Carrasco, ${ }^{2 *} \odot$ Josefa Tolosa, ${ }^{2} \odot$ Giulia Graziani, ${ }^{1}$ Anna Gaspari, ${ }^{1}$ \\ and Alberto Ritieni ${ }^{1}$ \\ ${ }^{1}$ Department of Pharmacy, Faculty of Pharmacy, University of Naples “Federico II," Via Domenico Montesano 49, 80131 Napoli, Italy \\ ${ }^{2}$ Laboratory of Food Chemistry and Toxicology, Faculty of Pharmacy, University of Valencia, Av. Vicent Andrés Estellés s/n, \\ 46100 Burjassot, Spain
}

\section{ABSTRACT}

Milk is a nutritious food suitable for infants and adults, and it plays an important role in the human diet. However, it may also be a vehicle for food contaminants. In this report, we developed a method using ultra-high-performance liquid chromatography coupled with high-resolution mass spectrometry (UHPLC-QExactive Orbitrap HRMS; Thermo Fisher Scientific, Waltham, MA) for simultaneous identification of target pharmacologically active substances and mycotoxins in milk. We also used the Q-Orbitrap operating in full scan mode to identify other possible drugs and microbial metabolites that occurred in samples. Fifty-six commercially available milk samples from the Italian market were analyzed. Investigated analytes were extracted using a QuEChERS (quick, easy, cheap, effective, rugged, and safe) approach. Method detection and quantification limits and performance criteria set by European regulations were fulfilled. Pharmacologically active substances were detected in $49 \%$ of samples (range 0.007-4.53 ng/mL), including nontarget mycotoxins. Retrospective analysis allowed us to identify other antibiotics and pharmacologically active substances, as well as nonregulated fungal/bacterial metabolites at a relatively high incidence. From the obtained values, the need for continuous monitoring of contaminants in the milk production chain is clear. This is the first study to assess the presence of pharmacologically active substances, mycotoxins, and other microbial metabolites in Italian milk samples using the UHPLC-Q-Orbitrap HRMS system.

Received July 16, 2019.

Accepted October 4, 2019.

*Corresponding author: yelko.rodriguez@uv.es
Key words: pharmacologically active substances, mycotoxins, milk, QuEChERS, ultra-highperformance liquid chromatography/high-resolution mass spectrometry (UHPLC-Q-Orbitrap HRMS), retrospective analysis

\section{INTRODUCTION}

Milk is widely consumed around the world and provides high-quality nutrition to infants, children, and adults because of its high macro- and micronutrient contents. According to the latest data from the Food and Agriculture Organization (FAO), annual per capita consumption of milk and dairy products for Europeans is $236.4 \mathrm{~kg}$, significantly higher than in other regions (United States $168.8 \mathrm{~kg}$, Asia $60.3 \mathrm{~kg}$, Africa $43.8 \mathrm{~kg}$; FAO, 2013). However, despite the benefits of milk from a nutritional point of view, it may also be a vehicle for food contaminants, such as mycotoxins and pharmacologically active substances as a result of improper agriculture practices or use of these drugs.

Mycotoxins are secondary toxic metabolites produced mainly by fungi belonging to the Aspergillus, Penicillium, Fusarium, Alternaria, and Claviceps genera. These fungi can colonize agricultural crops and produce mycotoxins during pre- and post-harvest practices, processing, and storage (Marin et al., 2013; Ogunade et al., 2018). When animals are given feed contaminated with mycotoxins, these toxic compounds are metabolized and transferred to animal-derived food, including milk (Negash, 2018). Mycotoxins may cause different toxic effects in animals and humans, including carcinogenic, mutagenic, teratogenic, immunotoxic, and estrogenic effects (Gallo et al., 2015). Among the mycotoxins identified so far, aflatoxins (AF) are the most extensively studied, and one of the most important classes from a public health perspective, because they have been 
classified as carcinogenic to humans by the International Agency for Research on Cancer (IARC, 2012). Other mycotoxins, such as ochratoxin A, zearalenone, fumonisins, and trichothecenes and their metabolites, have also been identified in milk samples and dairy products worldwide (Becker-Algeri et al., 2016; Benkerroum, 2016).

Milk may also contain pharmacologically active substances derived from the treatment of various animal diseases. Pharmacologically active substances are widely prescribed for both prophylactic and therapeutic reasons, and their use is permitted in veterinary practice to treat bacterial infections in livestock. Nowadays, pressure is growing, especially in Europe, to decrease or eliminate the use of antimicrobials for prophylaxis and growth promotion (Speksnijder and Wagenaar, 2018). Furthermore, as of 2022, a new European law will ban the prophylactic use of antibiotics in farming to reduce antibiotic resistance, in accordance with the World Health Organization recommendation (WHO, 2017).

The most commonly used pharmacologically active substances in food-producing animals are antimicrobial drugs, anti-inflammatory drugs (nonsteroidal antiinflammatory drugs, corticosteroids), and hormones. Antimicrobial drugs are the most contentious for their residues, which can occur in milk because of their high frequency of use (Morley et al., 2005; Baynes et al., 2016; Masiá et al., 2016). They can be classified into 5 major groups: tetracyclines, sulfonamides, aminoglycosides, macrolides, and $\beta$-lactams. Nonsteroidal anti-inflammatory drugs and corticosteroids also play a significant role, because they are highly effective in suppressing or preventing inflammation. Growth promoters (hormones) are also widely used to enhance milk production in cows and maintain production at bulk levels (Stolker and Brinkman, 2005). However, inappropriate use of pharmacologically active substances, or lack of respect for the withdrawal period, can lead to residues in animal-derived products, such as milk. In this way, pharmacologically active substances may enter the food chain and human exposure can induce acute poisoning, allergic reactions, or antibiotic resistance (Singer et al., 2017). Based on sales data published by the Ministry of Health, updated to 2016, the most commonly used veterinary drugs in Italy were penicillins, tetracyclines, and sulfonamides, which together represented $68.7 \%$ of total sales in 2016 (Ministero della salute, 2016). It should be noted that the use of antimicrobials on dairy farms varies by country. Serraino et al. (2013), who conducted a survey on antimicrobial use in dairy farms in the Emilia Romagna region of Italy, reported that the most commonly used antimicrobial agents belonged to the following classes: lactams $(51.3 \%$ of total use), fluoroquinolones (8.8\%), macrolides $(4,6 \%)$, sulfonamides $(3.1 \%)$, tetracyclines $(2.6 \%)$, lincosamides $(2.1 \%)$, and amphenicols $(0.3 \%)$; other antimicrobial agents used that did not belong to the above classes were rifaximin, clavulanic acid, and trimethoprim (12.4, 11.2, and $0.5 \%$, respectively). Among the wide range of pharmacologically active substances permitted in milk, special focus should be paid to substances with an extremely low maximum residue limit (MRL), or to substances that are prohibited in milk in accordance with current regulations.

To protect consumer health, the European Commission (EC) set MRL for pharmacologically active substances and maximum limits for mycotoxins in Commission Regulation (EC) No. 37/2010 and Commission Regulation (EC) No. 1881/2006, respectively. The MRL for pharmacologically active substances in milk range from 0.05 to $1,500 \mu \mathrm{g} / \mathrm{kg}$. For mycotoxins, $\mathrm{AFM}_{1}$, the principal hydroxylated $\mathrm{AFB}_{1}$ metabolite, is the only mycotoxin regulated for animal-derived products to date. The MRL of $\mathrm{AFM}_{1}$ in raw milk, heat-treated milk, and milk for the manufacture of milk-based products is $0.05 \mu \mathrm{g} / \mathrm{kg}$, and is restricted to $0.025 \mu \mathrm{g} / \mathrm{kg}$ for infant formulas and follow-on formulas, including infant milk and follow-on milk.

Until now, few methods have been proposed for the simultaneous identification of pharmacologically active substances and mycotoxins in food (Capriotti et al., 2012; Frenich et al., 2014; Danezis et al., 2016). Milk is a complex matrix that requires appropriate sample preparation. The QuEChERS approach (quick, easy, cheap, effective, rugged, and safe) has demonstrated many advantages over traditional extraction procedures (e.g., liquid-liquid extraction or solid-phase extraction), including speed, use of smaller quantities of organic solvents, and satisfactory recoveries obtained for a wide range of compounds (Rejczak and Tuzimski, 2015; Zhang et al., 2015; Rossi et al., 2018; Samsidar et al., 2018).

Ultra-high-performance liquid chromatography (UHPLC) has overcome the limitations of conventional HPLC (e.g., lower separation capacity and speed of analysis), improving sensitivity and resolution using packing materials with smaller particle size $(<2 \mu \mathrm{m}$; Fekete et al., 2014; Romera et al., 2018). Although tandem mass spectrometry (MS/MS) provides adequate quantification and high efficiency for multi-residue analyses, this strategy is sometimes limited for analysis at trace levels in complex matrices. High-resolution mass spectrometry (HRMS) using Orbitrap technology (Thermo Fisher Scientific, Waltham, MA) has made it possible to achieve high resolution and good specificity because of the mass accuracy provided by 
the HRMS detector, combined with traditional information (Kaufmann, 2014). This technique also enables the retrospective analysis of samples, in contrast to MS/MS.

The aim of the present study was (1) to develop a fast, cheap, robust, and reliable multi-residue method for the identification of target mycotoxins and pharmacologically active substances in milk from a QuEChERS-based extraction coupled to UHPLC Q-Orbitrap HRMS; (2) to apply the developed method and evaluate the occurrence of compounds described above in 56 samples collected from the Italian market; and (3) to detect possible nontargeted pharmacologically active substances and microbial metabolites in samples.

\section{MATERIALS AND METHODS}

\section{Chemicals and Materials}

Methanol, ethanol, dimethyl sulfoxide, acetonitrile, and water (LC-MS grade) were acquired from Merck (Darmstadt, Germany). Formic acid (MS grade) and ammonium formate (analytical grade) were purchased from Fluka (Milan, Italy). Syringe filters with a polytetrafluoroethylene membrane $(15 \mathrm{~mm} ; 0.22-\mu \mathrm{m}$ diameter; Sigma-Aldrich, Milan, Italy) were supplied by Phenomenex (Castel Maggiore, Italy). Glass amber vials with septum screw caps were supplied by Agilent Technologies (Milan, Italy). Sodium chloride, anhydrous sulfate sodium and anhydrous sodium acetate were from Sigma-Aldrich. Primary secondary amine sorbent and C18 (analytical grade) were from Supelco (Milan, Italy).

Standards of pharmacologically active substances and mycotoxins (purity $>98 \%$ ) were purchased from SigmaAldrich. Pharmacologically active substances were selected from those with lower MRL or prohibited in milk in accordance with EC Regulation No. 37/2010. The acquired pharmacologically active substances $(\mathrm{n}=24)$ standards were as follows: metronidazole, clenbuterol hydrochloride, meloxicam, dexamethasone, amoxicillin, ampicillin, procaine benzylpenicillin, ceftiofur, danofloxacin, monensin sodium, sulfadimidine, dapsone, chloramphenicol, abamectin, amitraz, cyhalothrin, cypermethrin, trichlorfon, colchicine, imidocarb, doramectin, ivermectin, eprinomectin, and deltamethrin.

The acquired mycotoxin $(\mathrm{n}=30)$ standards were as follows: aflatoxins $\left(\mathrm{AFM}_{1}, \mathrm{AFB}_{1}, \mathrm{AFB}_{2}, \mathrm{AFG}_{1}\right.$, and $\mathrm{AFG}_{2}$ ), ochratoxin $\mathrm{A}$, fumonisins $\left(\mathrm{FB}_{1}\right.$ and $\mathrm{FB}_{2}$ ), deoxynivalenol, 3-acetyldeoxynivalenol, 15-acetyldeoxynivalenol, HT-2 toxin, T-2 toxin, neosolaniol, diacetoxyscirpenol, fusarenon-X, zearalenone, $\alpha$-zearalenol, $\beta$-zearalenol, $\alpha$-zearalanol, $\beta$-zearalanol, zearalanone, beauvericin, enniatins $\left(A, A_{1}, B\right.$, and $\left.B_{1}\right)$, alternariol, and alternariolmonomethyl ether.

Individual standard solutions were prepared by diluting $1 \mathrm{mg}$ of each analyte in $1 \mathrm{~mL}$ of solvent (methanol, ethanol, acetonitrile, or dimethyl sulfoxide) based on the supplier's specifications. Three stock solutions were prepared: stock solution 1, including all mycotoxins except aflatoxins; stock solution 2, including aflatoxins, clenbuterol hydrochloride, and prohibited substances in milk; and stock solution 3, including the rest of the pharmacologically active substances. These stock solutions were prepared by taking a defined volume from each of the individual standard solutions and performing adequate dilution to reach appropriate working standard solutions for spiking experiments. The working standard solutions were prepared at 25, 250, and $1,250 \mathrm{ng} / \mathrm{L}$ from stock solution 1 ; at 2.5, 5, and $10 \mathrm{ng} / \mathrm{L}$ from stock solution 2 ; and at $0.2,2$, and $10 \mathrm{mg} / \mathrm{L}$ from stock solution 3 . All solutions were stored at $-20^{\circ} \mathrm{C}$ in screw-capped glass vials (Verex Vial; Phenomenex)

\section{Sampling}

Fifty-six commercially available milk samples were randomly purchased between October and November 2018 from different supermarkets in the Campania region of southern Italy. All purchased samples were UHT-type and classified as whole milk $(\mathrm{n}=20)$, semiskim milk $(\mathrm{n}=20)$, or skim milk $(\mathrm{n}=16)$. Samples were shipped to the laboratory in their original packages and stored at $4^{\circ} \mathrm{C}$ until analysis. Milk analysis was carried out within $2 \mathrm{~d}$ after arrival of the samples.

\section{Sample Preparation}

Samples were prepared as described by RodríguezCarrasco et al. (2018). Briefly, $10 \mathrm{~mL}$ of the sample was introduced into a 50-mL Falcon tube (Conical Polypropylene Centrifuge Tube; Thermo Fisher Scientific, Milan, Italy) and $2.5 \mathrm{~mL}$ of distilled water and $5 \mathrm{~mL}$ of acetonitrile containing 3.35\% formic acid ( $\mathrm{vol} / \mathrm{vol}$ ) were added. The sample was vortexed (ZX3; VEPL Scientific, Usmate, Italy) for $1 \mathrm{~min}$ and sonicated (LBS 1; Zetalab srl, Padua, Italy) for 15 min (vortexed at 5 -min intervals). Then, $4.0 \mathrm{~g}$ of anhydrous sulfate sodium, $1.2 \mathrm{~g}$ of sodium chloride, and $0.5 \mathrm{~g}$ of anhydrous sodium acetate were added, and the tube was shaken by hand for $1 \mathrm{~min}$ and then centrifuged (X3R Heraeus Multifuge; Thermo Fisher Scientific) for 3 min at 1,792 $\times g$ and $4^{\circ} \mathrm{C}$. The supernatant $(3 \mathrm{~mL})$ was collected into a $15-\mathrm{mL}$ Falcon tube containing $300 \mathrm{mg}$ of $\mathrm{C} 18$ sorbent, $140 \mathrm{mg}$ of primary secondary amine, and 1.5 $\mathrm{g}$ of anhydrous sulfate sodium. Then, the mixture was 
vortexed for $1 \mathrm{~min}$ and centrifuged for $1 \mathrm{~min}$ at 252 $\times g$ and $4^{\circ} \mathrm{C}$. The supernatant $(2 \mathrm{~mL})$ was transferred into a new glass tube and dried under gentle nitrogen flow at $45^{\circ} \mathrm{C}$. Finally, the residue was reconstituted with $0.5 \mathrm{~mL}$ of methanol:water $(70: 30, \mathrm{vol} / \mathrm{vol})$, filtered through a $0.22-\mu \mathrm{m}$ filter, and $5 \mu \mathrm{L}$ was injected into the UHPLC-Q-Orbitrap HRMS instrument.

\section{UHPLC-Q-Orbitrap HRMS Analysis}

Analyses were performed using an UHPLC instrument (Dionex Ultimate 3000; Thermo Fisher Scientific, Waltham, MA) coupled to a Q-Exactive Orbitrap mass spectrometer (Thermo Fisher Scientific). The UHPLC system consisted of a degassing system, a quaternary UHPLC pump working at 1,250 bar (125 MPa), an autosampler device, and a thermostatically controlled Luna Omega column $(50 \times 2.1 \mathrm{~mm}, 1.6 \mu \mathrm{m}$; Phenomenex) held at $30^{\circ} \mathrm{C}$. The mobile phase consisted of (A) water with $0.1 \%$ formic acid containing $5 \mathrm{mM}$ ammonium formate and (B) methanol with $0.1 \%$ formic acid containing $5 \mathrm{mM}$ ammonium formate. A linear gradient elution program was applied as follows: an initial $0 \%$ B was held for $1 \mathrm{~min}$, increased to $95 \%$ B over $1 \mathrm{~min}$, and held for $0.5 \mathrm{~min}$. Then, the gradient was linearly decreased to $75 \%$ over $2.5 \mathrm{~min}$, and decreased again to $60 \%$ B over 1 min. Finally, the gradient was reduced to $0 \%$ over $0.5 \mathrm{~min}$ and held for $1.5 \mathrm{~min}$ for re-equilibration, for a total run time of $8 \mathrm{~min}$. The flow rate was $0.4 \mathrm{~mL} / \mathrm{min}$.

Detection was performed using a Q-Exactive Orbitrap mass spectrometer. The mass spectrometer was operated in positive and negative ion mode by setting 2 scan events: full ion MS and data-dependent fragmentation $\left(\mathbf{d d}-\mathbf{M S}^{\mathbf{2}}\right)$. Full scan data were acquired at a resolving power of 70,000 full width at half maximum at $200 \mathrm{~m} / z$. Mass range in the full scan experiments was set at 100 to $1,000 \mathrm{~m} / z$. The conditions in positive ionization mode $\left(\mathbf{E S I}^{+}\right)$were as follows: spray voltage 4,000 V; capillary temperature $290^{\circ} \mathrm{C}$; S-lens RF level 50 ; sheath gas pressure $\left(\mathrm{N}_{2}>95 \%\right) 35$; auxiliary gas $\left(\mathrm{N}_{2}>95 \%\right) 10$; and auxiliary gas heater temperature $305^{\circ} \mathrm{C}$. The conditions in negative ionization mode $\left(\mathbf{E S I}^{-}\right)$were as follows: spray voltage $4,000 \mathrm{~V}$; capillary temperature $290^{\circ} \mathrm{C}$; S-lens RF level 50; sheath gas pressure $\left(\mathrm{N}_{2}>95 \%\right) 35$; auxiliary gas $\left(\mathrm{N}_{2}>95 \%\right) 10$; and auxiliary gas heater temperature $305^{\circ} \mathrm{C}$. The parameters for the scan event of dd-MS ${ }^{2}$ were as follows: mass resolving power of 17,500 full width at half maximum $(200 \mathrm{~m} / \mathrm{z})$, AGC target at $2 \times 10^{5}$, maximum IT at 50 $\mathrm{ms}$, and isolation window at $2.0 \mathrm{~m} / z$ for an accurate mass measurement fragments (Rodríguez-Carrasco et al., 2018).
Screening was carried out by investigating spectral data collected using the following: a mycotoxin spectral library (version 1.1 for Library View Software, AB Sciex, Framingham, MA) containing spectral data for 245 mycotoxins and other fungal/bacterial metabolites and 236 full MS/MS spectral library entries; and a veterinary drugs spectral library (antibiotics spectral library version 1.0 for Library View Software, AB Sciex) containing 244 antibiotic and veterinary drugs and 259 high-resolution MS/MS spectral library entries. For accurate mass measurement, we performed identification and confirmation at a mass tolerance of $5 \mathrm{ppm}$ for the molecular ion and for both fragments. Precursor and product ions were required to identify both targeted and nontargeted pharmacologically active substances. The Q-Orbitrap Full MS/dd-MS ${ }^{2}$ (data-dependent acquisition) was used to acquire product-ion spectra of individual veterinary drugs to build a compound database and a mass spectral library. The in-spectrum mass correction or solvent background lock-mass correction was used to minimize mass error when building the compound database from experimental dd- $\mathrm{MS}^{2}$ accurate mass data. Retention time alignment and response threshold adjustment were used to eliminate or reduce false negatives and false positives (Romera et al., 2018). Data analysis and processing were conducted using Quan/Qual Browser Xcalibur software, version 3.1.66 (Thermo Fisher Scientific).

\section{Validation of the Method}

Our method was validated in-house according to European regulations (EC 2002/657, EC 401/2006, SANTE 12089/2016). The parameters evaluated were linearity, matrix effect, specificity, trueness, precision, and sensitivity. All parameters were assessed in triplicate. For confirmation criteria, we compared the retention times of analytes in standards and samples at a tolerance of $\pm 2.5 \%$. Data quality was monitored using a comprehensive range of quality assurance and quality control procedures. We included a rigorous and systematic control, a reagent blank, a procedural blank, a replicate sample, and a matrix-matched calibration in each batch of samples. We used 3 spiking levels for each stock solution for analytical quality control.

\section{Statistical Analysis}

All method performance experiments were conducted in triplicate, and data are expressed as mean \pm relative standard deviation (RSD). Statistical analysis was performed using Student's $t$ test. Comparisons at $P \leq$ 0.05 were considered statistically significant. 


\section{RESULTS AND DISCUSSION}

\section{Optimization of the UHPLC-Q-Orbitrap HRMS Conditions}

We obtained full scan and MS/MS spectra of the 54 analytes by direct infusion of each compound at $1 \mu \mathrm{g} / \mathrm{mL}$ into the Q-Orbitrap system using a syringe injection at a flow rate of $8 \mu \mathrm{L} / \mathrm{min}$. Experiments were achieved in both $\mathrm{ESI}^{+}$and $\mathrm{ESI}^{-}$modes. Most of the studied analytes exhibited better fragmentation patterns in $\mathrm{ESI}^{+}$mode and predominantly produced the quasi molecular ion $[\mathrm{M}+\mathrm{H}]^{+}$. After full scan analysis, we listed the accurate mass of the characteristic ions (precursor ions) in an inclusion list.

In the current study, we used the UHPLC Luna Omega column $(50 \times 2.1 \mathrm{~mm}, 1.6 \mu \mathrm{m}$; Phenomenex $)$ and tested several gradient profiles to achieve good chromatographic separation of the studied compounds. The tested gradients were as follows:

- Gradient 1: Initial 10\% B was held for 2 min, then increased to $100 \%$ B over 3 min, and held for 1.5 min. Then, the gradient was linearly decreased to $10 \% \mathrm{~B}$ over $1 \mathrm{~min}$ and held for $2 \mathrm{~min}$ for reequilibration, giving a total run time of $9.5 \mathrm{~min}$.

- Gradient 2: Initial 5\% B was held for $1 \mathrm{~min}$, then increased to $95 \%$ B over $1.5 \mathrm{~min}$, held for $1 \mathrm{~min}$, and decreased again to $70 \% \mathrm{~B}$ over $2 \mathrm{~min}$. Then, the gradient was linearly decreased to $5 \%$ B over 1 min and held for 2 min for re-equilibration, giving a total run time of 8.5 min.

- Gradient 3: Initial 0\% B was held for 1 min, then increased to $95 \%$ B over $1 \mathrm{~min}$, and held for 0.5 min. Then, the gradient was linearly decreased to $75 \%$ over $2.5 \mathrm{~min}$, and decreased again to $60 \%$ $\mathrm{B}$ over $1 \mathrm{~min}$. Finally, the gradient was reduced to $0 \%$ over $0.5 \mathrm{~min}$ and held for $1.5 \mathrm{~min}$ for reequilibration, giving a total run time of $8 \mathrm{~min}$.

Some analytes were not retained when using gradient 1 conditions. With gradient 2 , all target compounds showed retention times between 2.5 and $7 \mathrm{~min}$, but the peak response for some was irregular. For gradient 3, we obtained good separation and peak shape for all target analytes (Supplemental Table S1; https://doi .org/10.3168/jds.2019-17277).

\section{Optimization of the Sample Preparation Procedure}

Sample preparation is a critical step in multi-residual identification because of the different physicochemical properties of the compounds, the different concentra- tions of the investigated analytes, or the complexity of the matrix, among others (Souza-Silva et al., 2015; Kiljanek et al., 2016; Mainero Rocca et al., 2017).

To date, limited information has been reported in the literature about the simultaneous identification of pharmacologically active substances and mycotoxins in milk, as shown in Table 1.

In this work, we used a QuEChERS-based procedure for the extraction of mycotoxins and pharmacologically active substances in milk as a starting point, with minor modifications (Zhou et al., 2018).

We evaluated critical extraction parameters such as sample:solvent ratio and sonication time. First, we tested 3 different sample:solvent ratios (A: $5 \mathrm{~mL}$ sample:10 mL solvent; B: $5 \mathrm{~mL}$ sample: $5 \mathrm{~mL}$ solvent; C: $10 \mathrm{~mL}$ sample: $5 \mathrm{~mL}$ solvent) by preparing the abovementioned ratios in triplicate in samples spiked at 0.5 $\mu \mathrm{g} / \mathrm{L}$ (analytes included in stock 1 and stock 3) and $0.01 \mu \mathrm{g} / \mathrm{L}$ (analytes included in stock 2). The conditions for ratio A were not suitable to reach appropriate recovery values $(<40 \%)$. However, we did observe acceptable trueness and precision results with the conditions for ratio B (recovery range: $62-87 \%$; RSD $<11 \%$ ) and ratio $\mathrm{C}$ (recovery range: $72-93 \%$; RSD $<9 \%$ ). Even though more interference from the matrix was present in ratio $\mathrm{C}$ than in ratio $\mathrm{B}$, we observed moderate matrix effects $(>75 \%)$ for all studied compounds. The sensitivity of the method was also improved as a result of the concentration factor of ratio $\mathrm{C}$, reaching limits of quantification of 0.005 to $0.5 \mathrm{ng} / \mathrm{L}$.

To improve extraction efficiency, we tested different sonication times $(5,15,25 \mathrm{~min})$. We found no statistical differences $(P>0.05)$ between 15-min sonication (recovery range: $68-96 \%$; RSD $<12 \%$ ) and 25 -min sonication (recovery range: $73-98 \%$; RSD $<12 \%$ ); we obtained recoveries below $60 \%$ at 5 min sonication time. We selected $10 \mathrm{~mL}$ of sample: $5 \mathrm{~mL}$ extraction solvent (ratio C) and 15-min sonication time as the optimal conditions for simultaneous extraction of the studied analytes based on these results.

\section{Analytical Features of the Proposed Method}

Table 2 shows the performance of the optimized method. We performed calibration curves and matrixmatched calibrations of each analyte at 8 concentration levels, ranging from the limit of quantification (LOQ) to $100 \mu \mathrm{g} / \mathrm{L}$. Each calibration curve was prepared in triplicate. We obtained regression coefficients $>0.990$ for all studied compounds. Co-eluted matrix components can interfere with the ionization process, typically producing signal suppression. To evaluate this effect, we compared the matrix-matched calibration curves (A) 
IzZo et al.: SCREENING MILK FOR MYCOTOXINS

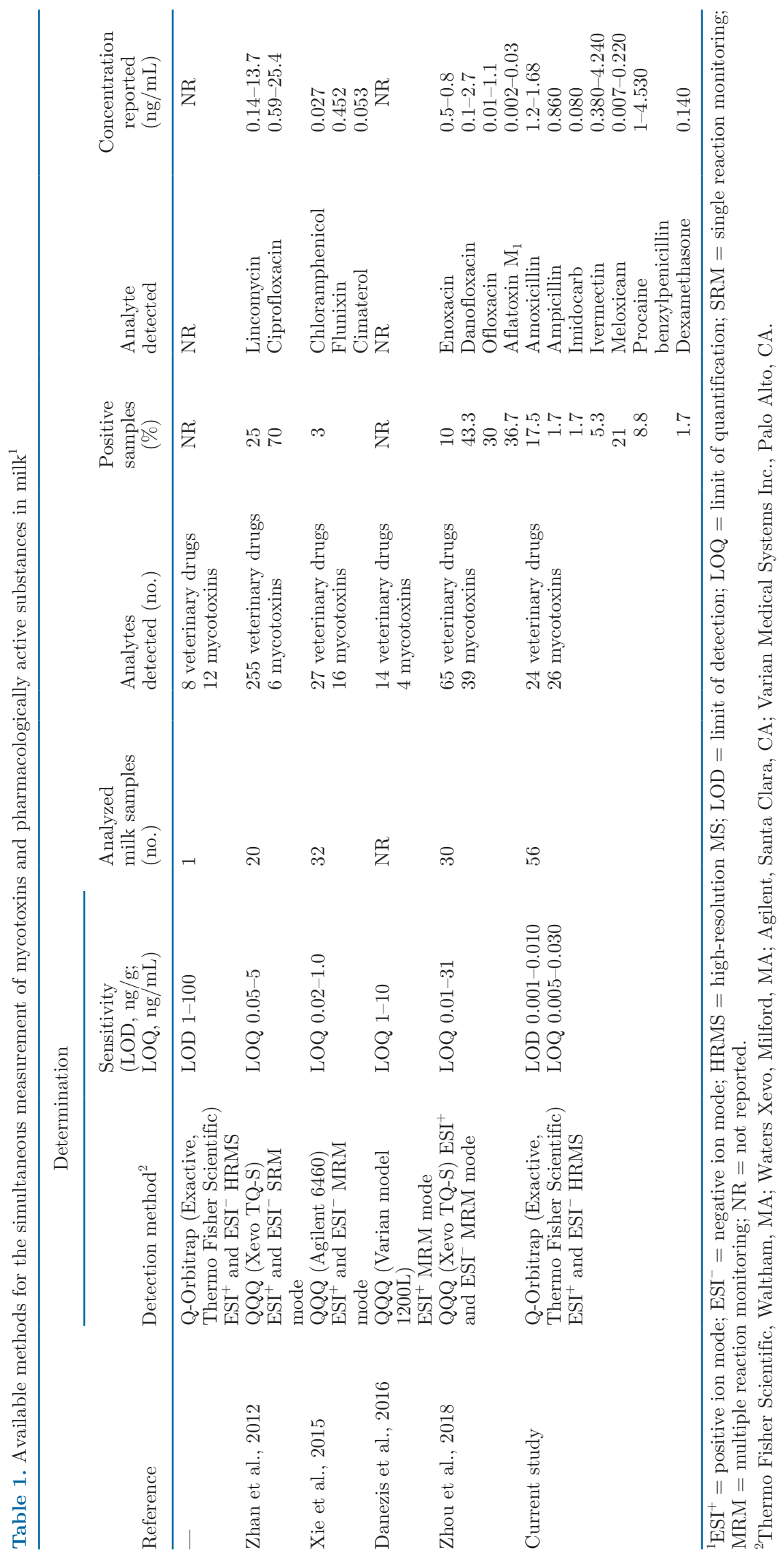


with the standard calibration curves (B) and expressed them as the ratio percentage between these slopes. Thus, the ratio $(\mathrm{A} / \mathrm{B} \times 100)$ was defined as the matrix effect (signal suppression enhancement; SSE, \%). An SSE of $100 \%$ meant that no matrix effect occurred. We found signal suppression if SSE was lower than $100 \%$ and signal enhancement if SSE was higher than 100\%. We obtained moderate matrix effects in the range of 75 to $97 \%$. We determined specificity by analyzing a blank milk extract 10 times to confirm the absence of possible sample interferences at the same retention time as the studied analytes. For all target analytes, we detected no significant interfering substances in their elution time.

We calculated method trueness, expressed as recovery data, at 3 spiking levels: $0.05,0.5$, and $2.5 \mu \mathrm{g} / \mathrm{L}$ for analytes included in stock solution $1 ; 0.005,0.01$, and $0.05 \mu \mathrm{g} / \mathrm{L}$ for analytes included in stock solution 2; and $0.5,5$, and $25 \mu \mathrm{g} / \mathrm{L}$ for analytes included in stock solution 3 . This method provided satisfactory recoveries at all spiking levels, ranging from 60 to $97 \%$. We evaluated the precision of the method in terms of repeatability (intra-day precision, $\mathrm{n}=3$ ) and reproducibility (inter-day precision, $\mathrm{n}=3$ ) in milk samples spiked at the levels above reported. The precision data, expressed as percent RSD, showed that the proposed method was repeatable $(\mathrm{RSD}<7 \%)$ and reproducible $(\mathrm{RSD}<14 \%)$. We determined sensitivity by calculating the limit of detection (LOD) and LOQ for each target compound. We established the LOD as the lowest concentration tested that allowed us to identify the molecular ion with a mass error $<5 \mathrm{ppm}$. We established LOQ as the lowest concentration of the analyte that generated a chromatographic peak with acceptable accuracy and precision results $(<20 \%)$. The LOD ranged from 0.001 to 0.010 ; the LOQ ranged from 0.005 to $0.030 \mathrm{ng} / \mathrm{mL}$. The sensitivity achieved with the proposed method allow us to quantify levels up to 10 times lower for mycotoxins compared to other published methods (Table 1).

\section{Target Mycotoxins and Pharmacologically Active Substances in Milk Samples}

Most of the studies evaluating the occurrence of mycotoxins in milk have focused on $\mathrm{AFM}_{1}$ only (Tsiplakou et al., 2014; Mwanza et al., 2015). However, over the past decade attention to human health risk has also been extended to other mycotoxins, because of cows' ability to metabolize these fungal toxic agents and transfer them to milk (Battacone et al., 2009; Gruber-Dorninger et al., 2017). As a result, we applied a validated multi-mycotoxin method to 56 milk samples acquired from different supermarkets in the Campania region in southern Italy. None of the 30 target my- cotoxins were detected in any analyzed milk sample. Different results were reported by Flores-Flores et al. (2015), who reviewed the occurrence of mycotoxins in milk samples worldwide. Ochratoxin A was found in $4.55 \%$ ( $\mathrm{n}=505)$ of Italian, Sweden, Norwegian, French, and Chinese bovine milk samples at concentrations of 5 to $84.1 \mathrm{ng} / \mathrm{L}$. The presence of FB1 was reported in $5.4 \%(\mathrm{n}=165)$ of Italian and American samples at concentrations of 260 to $1,290 \mathrm{ng} / \mathrm{L})$. Cyclopiazonic acid was detected in $7.35 \%(\mathrm{n}=68)$ at concentrations of 6,330 to $9,700 \mathrm{ng} / \mathrm{L}$, and zearalenone was found in $16 \%(\mathrm{n}=230)$ and $\alpha$-zearalanol in $0.9 \%(\mathrm{n}=103)$ of Chinese milk samples. Continuous monitoring of the levels of mycotoxins in milk is necessary.

In contrast, we found up to 7 pharmacologically active substances belonging to antibiotic drugs (amoxicillin, ampicillin, benzylpenicillin procaine), nonsteroidal anti-inflammatory drugs (meloxicam), corticosteroids (dexamethasone), antiprotozoans (imidocarb), and anthelmintics (ivermectin) in $49 \%$ of analyzed samples at concentrations ranging from 0.007 to $4.53 \mathrm{ng} / \mathrm{mL}$. Meloxicam was the most commonly detected pharmacologically active substance $(21 \%, \mathrm{n}=12)$, at concentrations of 0.007 to $0.220 \mathrm{ng} / \mathrm{mL}$. Despite its common presence, meloxicam was quantified at levels below its MRL $(15 \mu \mathrm{g} / \mathrm{kg})$. Only benzylpenicillin procaine was detected in 1 analyzed sample at a concentration slightly higher than its MRL $(4 \mathrm{ng} / \mathrm{mL})$. With respect to the occurrence of pharmacologically active substances in other studies, Nikolić et al. (2011) analyzed 6,161 milk samples from Montenegro and detected antibiotics residues in $7.8 \%$ of the samples. Similarly, Zhu et al. (2016) reported the presence of amoxicillin in 3\% of Chinese milk samples $(\mathrm{n}=33)$ at an average concentration of $22.9 \mathrm{ng} / \mathrm{mL}$.

Surprisingly, chloramphenicol, a prohibited substance, was detected in $63.6 \%$ of Croatian milk samples $(\mathrm{n}=1,259)$ at concentrations from 0.001 to $0.118 \mathrm{ng} /$ $\mathrm{mL}$ (Bilandžić et al., 2010) and in 3\% of Chinese milk samples ( $\mathrm{n}=32$; Xie et al., 2015). We detected no prohibited residues, including chloramphenicol, dapsone, metronidazole, or colchicine, in samples for the current study (LOQ $0.0005 \mu \mathrm{g} / \mathrm{L}$ ).

Of special toxicological concern is the co-occurrence of pharmacologically active substances in samples. Up to 3 different pharmacologically active substances were detected simultaneously in $7 \%$ of the analyzed milk samples. Even though the individual concentrations of the pharmacologically active substances in the samples were below the MRL, the possible adverse health effects from the additive or synergistic action of different chemical substances coexisting in samples should be considered in risk assessment studies. 
Table 2. Method performance: linearity, matrix effect (SSE \%), recovery (values indicate levels at which analytes were spiked), and LOQ; analyte groupings are as follows: stock solution 1 includes all mycotoxins except aflatoxins; stock solution 2 includes aflatoxins, clenbuterol hydrochloride, and substances prohibited in milk; and stock solution 3 includes the rest of the pharmacologically active substances ${ }^{1}$

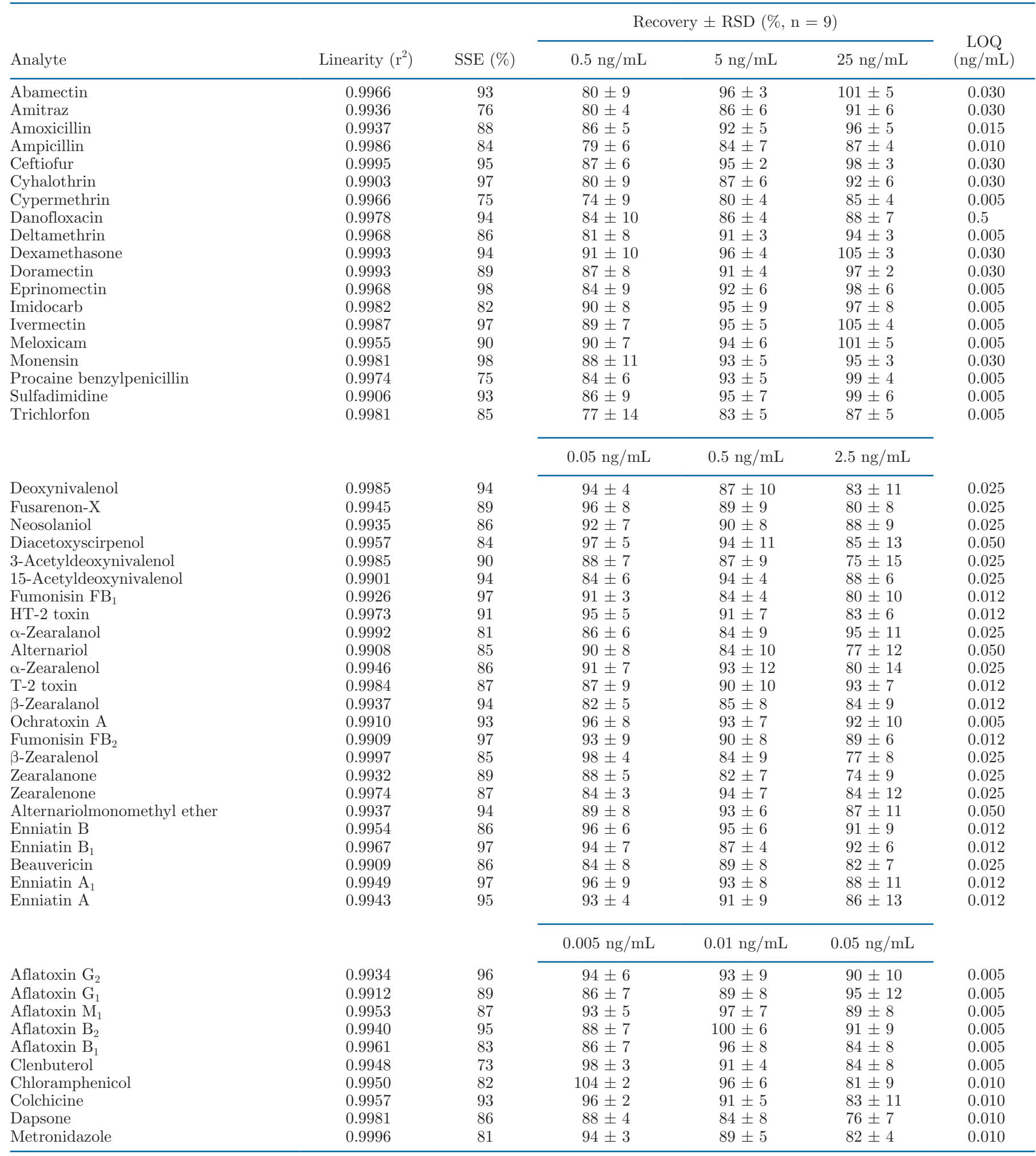

${ }^{1} \mathrm{SSE}=$ signal suppression enhancement; RSD = relative standard deviation; LOQ = limit of quantification. 


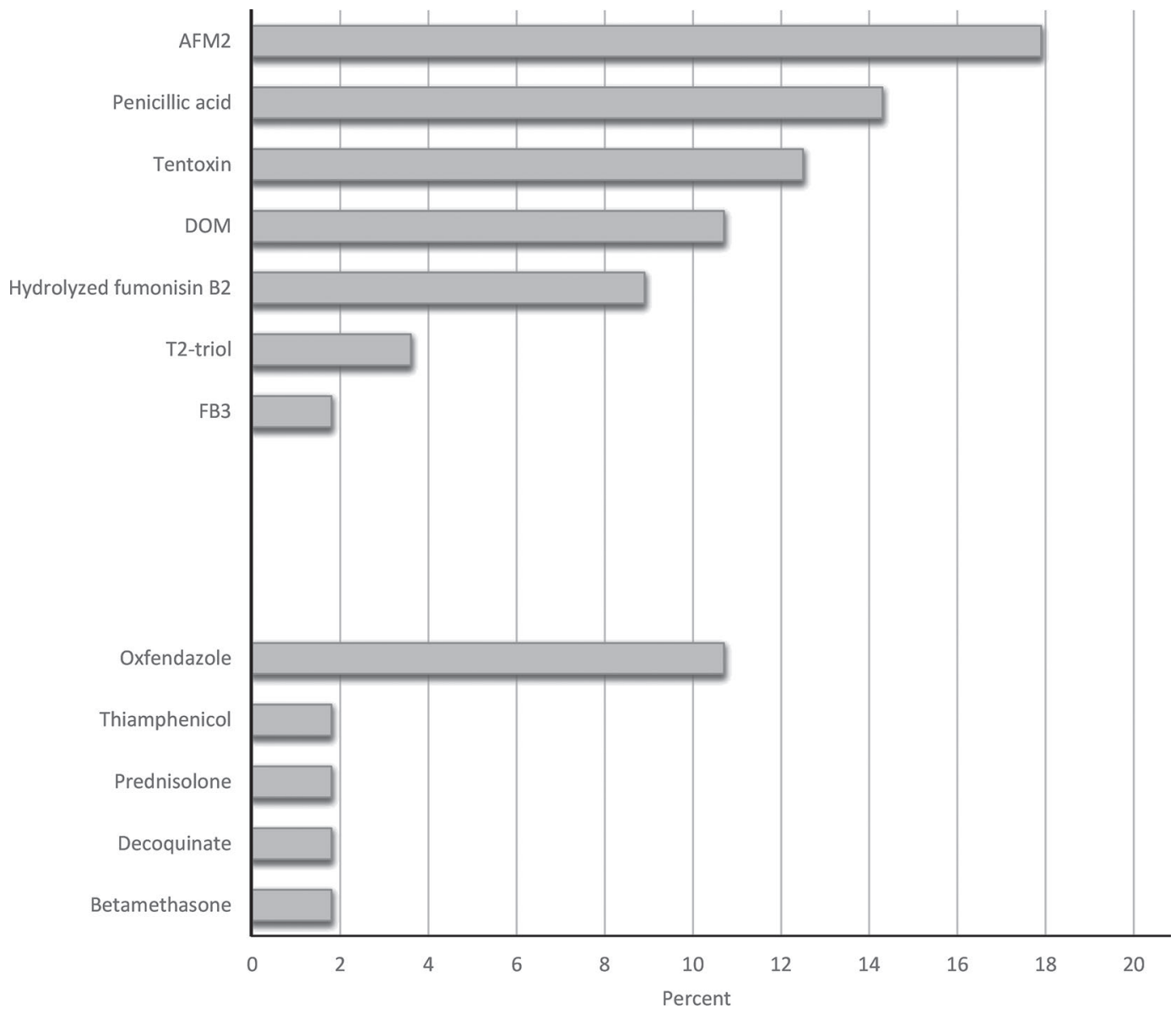

Figure 1. Results obtained from retrospective analysis of the data. AFM2 = aflatoxin $\mathrm{M}_{2} ; \mathrm{FB} 3=$ fumonisin $\mathrm{B}_{3} ; \mathrm{DOM}=$ deepoxy-deoxynivalenol. Percent $=$ occurrence of nontarget analytes in assayed milk samples (\%).

\section{Retrospective Screening Analysis of Real Samples}

The post-target screening approach enabled us to detect contaminants, including fungal/bacterial metabolites and pharmacologically active substances, in analyzed milk samples using spectral libraries. Results obtained are shown in Figure 1. We tentatively identified up to 7 mycotoxins and fungal metabolites. The presence of aflatoxin $\mathrm{AFM}_{2}$, the hydroxylated metabolite of $\mathrm{AFB}_{2}$, in 10 out of the 56 analyzed milk samples should be highlighted. For lactating ruminants, a substantial literature exists about the occurrence and transfer of $\mathrm{AFB}_{1}$ from feed into milk, but little is known about $\mathrm{AFB}_{2}$ or $\mathrm{AFM}_{2}$. Lee and Lee (2015) reported $\mathrm{AFM}_{2}$ contamination from 20.62 to $55.67 \mathrm{ng} / \mathrm{kg}$ in milk samples from Korea $(\mathrm{n}=100)$. Similar findings were reported in $48 \%$ of Japanese milk samples $(n=52)$ at concentrations of 0.003 to $0.0034 \mu \mathrm{g} / \mathrm{kg}$ (Nakajima et al., 2004). We also tentatively identified other Penicillium, Alternaria, or Fusarium metabolites in current study, including penicillic acid $(\mathrm{n}=8)$, tentoxin $(\mathrm{n}=$
7), deepoxy-deoxynivalenol $(\mathrm{n}=6)$, and hydrolyzed fumonisin $\mathrm{B}_{2}(\mathrm{n}=5)$, among others. These results could be justified when taking into account findings of Tozzi et al. (2016), who demonstrated that the bioconversion of mycotoxins by ruminants is higher than by monogastrics. Therefore, our findings point to the necessity of evaluating other fungal toxic metabolites in milk monitoring studies.

Retrospective analysis allowed us to tentatively identify oxfendazole (an anthelmintic) in 6 of the 56 analyzed milk samples. Similarly, oxfendazole was detected in 2 of 73 Greek milk samples at an average concentration of $1 \mu \mathrm{g} / \mathrm{kg}$ (Dasenaki and Thomaidis, 2015), far below the MRL for this benzimidazole in milk. We also identified other pharmacologically active substances, such as thiamphenicol, prednisolone, and betamethasone, in 1 milk sample (Figure 1).

Of special interest was the putative identification of decoquinate in 1 sample. Decoquinate was also reported in 1 of 120 analyzed Spanish milk samples, at a concentration of $5 \mu \mathrm{g} / \mathrm{kg}$ (Nebot et al., 2012). Although 
decoquinate was initially developed as an anticoccidial drug, it can improve milk production and weight gain and can reach high concentrations in milk. For this reason, the use of decoquinate is not permitted in animals that produce milk for human consumption, as stated in Commission Regulation (EC) No. 37/2010.

\section{CONCLUSIONS}

In the current study, we optimized an alternative analytical tool for the simultaneous identification of mycotoxins $(\mathrm{n}=30)$ and pharmacologically active substances $(\mathrm{n}=24)$ in milk based on QuEChERS-UHPLCQ-Orbitrap HRMS. We satisfactorily validated this method according to European Commission Decision 2002/657/EC, obtaining good results in terms of trueness, reproducibility, and repeatability; compared with previous methods, it was more sensitive, efficient, and quick (chromatography run time of $8 \mathrm{~min}$ ). We applied the developed methodology to 56 Italian milk samples. The results of the targeted analysis showed that no analyzed sample was contaminated with mycotoxins, but we did find pharmacologically active substances in $49 \%$ of samples, at concentrations of 0.007 to 4.53 $\mathrm{ng} / \mathrm{mL}$. Orbitrap-MS is useful for post-acquisition data processing, and the retrospective approach allowed the tentative identification of several fungal/bacterial metabolites, as well as other pharmacologically active substances, including those prohibited in milk. Because milk is their recommended food source, newborns and infants are exposed to these toxic compounds. Because their ability to metabolize these toxic agents has not yet fully developed, constant monitoring on dairy farms is needed to ensure food safety for consumers.

\section{ACKNOWLEDGMENTS}

The authors are grateful for the financial support given by Spanish Ministry of Economy and Competitiveness (AGL-2016-77610-R). The authors declare no conflict of interest.

\section{REFERENCES}

Battacone, G., A. Nudda, M. Palomba, A. Mazzette, and G. Pulina. 2009. The transfer of aflatoxin M1 in milk of ewes fed diet naturally contaminated by aflatoxins and effect of inclusion of dried yeast culture in the diet. J. Dairy Sci. 92:4997-5004. https://doi .org/10.3168/jds.2008-1684.

Baynes, R. E., K. Dedonder, L. Kissell, D. Mzyk, T. Marmulak, G. Smith, L. Tell, R. Gehring, J. Davis, and J. E. Riviere. 2016. Health concerns and management of select veterinary drug residues. Food Chem. Toxicol. 88:112-122. https://doi.org/10.1016/ j.fct.2015.12.020.

Becker-Algeri, T. A., D. Castagnaro, K. de Bortoli, C. de Souza, D. A. Drunkler, and E. Badiale-Furlong. 2016. Mycotoxins in bovine milk and dairy products: a review. J. Food Sci. 81:R544-R552. https://doi.org/10.1111/1750-3841.13204.

Benkerroum, N. 2016. Mycotoxins in dairy products: A review. Int. Dairy J. 62:63-75. https://doi.org/10.1016/j.idairyj.2016.07.002.

Bilandžić, N., I. Varenina, and B. Solomun. 2010. Aflatoxin M1 in raw milk in Croatia. Food Control 21:1279-1281. https://doi.org/10 .1016/j.foodcont.2010.03.003.

Capriotti, A. L., C. Cavaliere, S. Piovesana, R. Samperi, and A. Laganà. 2012. Multiclass screening method based on solvent extraction and liquid chromatography-tandem mass spectrometry for the determination of antimicrobials and mycotoxins in egg. J. Chromatogr. A 1268:84-90. https://doi.org/10.1016/j.chroma .2012.10.040.

Danezis, G. P., C. J. Anagnostopoulos, K. Liapis, and M. A. Koupparis. 2016. Multi-residue analysis of pesticides, plant hormones, veterinary drugs and mycotoxins using HILIC chromatographyMS/MS in various food matrices. Anal. Chim. Acta 942:121-138. https://doi.org/10.1016/j.aca.2016.09.011.

Dasenaki, M. E., and N. S. Thomaidis. 2015. Multi-residue determination of 115 veterinary drugs and pharmaceutical residues in milk powder, butter, fish tissue and eggs using liquid chromatographytandem mass spectrometry. Anal. Chim. Acta 880:103-121. https: //doi.org/10.1016/j.aca.2015.04.013.

Fekete, S., J. Schappler, J. L. Veuthey, and D. Guillarme. 2014. Current and future trends in UHPLC. Trends Anal. Chem. 63:2-13. https://doi.org/10.1016/j.trac.2014.08.007.

Flores-Flores, M. E., E. Lizarraga, A. López de Cerain, and E. González-Peñas. 2015. Presence of mycotoxins in animal milk: A review. Food Control 53:163-176. https://doi.org/10.1016/j .foodcont.2015.01.020.

FAO (Food and Agriculture Organization). 2013. Food Balance Sheet. Accessed Apr. 17, 2019. http://www.fao.org/faostat/en/\#data/ FBS.

Frenich, A. G., R. Romero-González, and M. del Mar Aguilera-Luiz. 2014. Comprehensive analysis of toxics (pesticides, veterinary drugs and mycotoxins) in food by UHPLC-MS. Trends Anal. Chem. 63:158-169. https://doi.org/10.1016/j.trac.2014.06.020.

Gallo, A., G. Giuberti, J. Frisvad, T. Bertuzzi, and K. Nielsen. 2015 Review on mycotoxin issues in ruminants: Occurrence in forages, effects of mycotoxin ingestion on health status and animal performance and practical strategies to counteract their negative effects. Toxins (Basel) 7:3057-3111. https://doi.org/10.3390/ toxins7083057.

Gruber-Dorninger, C., B. Novak, V. Nagl, and F. Berthiller. 2017. Emerging mycotoxins: Beyond traditionally determined food contaminants. J Agric. Food Chem. 65:7052-7070. https://doi.org/10 .1021/acs.jafc.6b03413.

IARC (International Agency for Research on Cancer). 2012. Agents Classified by the IARC Monographs. Vol. 1 IARC, Lyon, France.

Kaufmann, A. 2014. Combining UHPLC and high-resolution MS: A viable approach for the analysis of complex samples? Trends Anal. Chem. 63:113-128. https://doi.org/10.1016/j.trac.2014.06.025.

Kiljanek, T., A. Niewiadowska, S. Semeniuk, M. Gaweł, M. Borzęcka, and A. Posyniak. 2016. Multi-residue method for the determination of pesticides and pesticide metabolites in honeybees by liquid and gas chromatography coupled with tandem mass spectrometry-honeybee poisoning incidents. J. Chromatogr. A 1435:100114. https://doi.org/10.1016/j.chroma.2016.01.045.

Lee, D., and K. G. Lee. 2015. Analysis of aflatoxin M1 and M2 in commercial dairy products using high-performance liquid chromatography with a fluorescence detector. Food Control 50:467-471. https://doi.org/10.1016/j.foodcont.2014.09.020.

Mainero Rocca, L., A. Gentili, V. Perez-Fernandez, and P. Tomai. 2017. Veterinary drugs residues: A review of the latest analytical research on sample preparation and LC-MS based methods. Food Addit. Contam. Part A. Chem. Anal. Control Expo. Risk Assess. 34:766-784. https://doi.org/10.1080/19440049.2017.1298846.

Marin, S., A. J. Ramos, G. Cano-Sancho, and V. Sanchis. 2013. Mycotoxins: Occurrence, toxicology, and exposure assessment. Food Chem. Toxicol. 60:218-237. https://doi.org/10.1016/j.fct.2013.07 .047 . 
Masiá, A., M. M. Suarez-Varela, A. Llopis-Gonzalez, and Y. Pico. 2016. Determination of pesticides and veterinary drug residues in food by liquid chromatography-mass spectrometry: A review. Anal. Chim. Acta 936:40-61. https://doi.org/10.1016/j.aca.2016 .07 .023 .

Ministero della salute. 2016. Dati di vendita dei medicinali veterinari contenenti agenti antimicrobici. Trend in Italia Anno 2016. Accessed Oct. 23, 2019. http://www.salute.gov.it/imgs/C_17 _pubblicazioni_2780_allegato.pdf

Morley, P. S., M. D. Apley, T. E. Besser, D. P. Burney, P. J. FedorkaCray, M. G. Papich, J. L. Traub-Dargatz, and J. S. Weese. 2005. Antimicrobial drug use in veterinary medicine. J. Vet. Intern. Med. 19:617-629. https://doi.org/10.1111/j.1939-1676.2005.tb02739.x.

Mwanza, M., A. Abdel-Hadi, A. M. Ali, and M. Egbuta. 2015. Evaluation of analytical assays efficiency to detect aflatoxin M1 in milk from selected areas in Egypt and South Africa. J. Dairy Sci. 98:6660-6667. https://doi.org/10.3168/jds.2014-9220.

Nakajima, M., S. Tabata, H. Akiyama, Y. Itoh, T. Tanaka, H. Sunagawa, T. Tyonan, T. Yoshizawa, and S. Kumagai. 2004. Occurrence of aflatoxin M1 in domestic milk in Japan during the winter season. Food Addit. Contam. 21:472-478. https://doi.org/10.1080/ 02652030410001677817.

Nebot, C., A. Iglesias, P. Regal, J. M. Miranda, C. Fente, and A. Cepeda. 2012. A sensitive and validated HPLC-MS/MS method for simultaneous determination of seven coccidiostats in bovine whole milk. Food Control 27:29-36. https://doi.org/10.1016/j .foodcont.2012.02.012.

Negash, D. 2018. A review of aflatoxin: occurrence, prevention, and gaps in both food and feed safety. J. Appl. Microbiol. 8:35-43. https://doi.org/10.15406/jnhfe.2018.08.00268

Nikolić, N., S. Mirecki, and M. Blagojević. 2011. Presence of inhibitory substances in raw milk in the area of Montenegro. Mljekarstvo 61:182-187.

Ogunade, I. M., C. Martinez-Tuppia, O. C. M. Queiroz, Y. Jiang, P. Drouin, F. Wu, D. Vyas, and A. T. Adesogan. 2018. Silage review: Mycotoxins in silage: Occurrence, effects, prevention, and mitigation. J. Dairy Sci. 101:4034-4059. https://doi.org/10.3168/ jds.2017-13788.

Rejczak, T., and T. Tuzimski. 2015. A review of recent developments and trends in the QuEChERS sample preparation approach. Open Chem. 13:980-1010. https://doi.org/10.1515/chem-2015-0109.

Rodríguez-Carrasco, Y., L. Izzo, A. Gaspari, G. Graziani, J. Mañes, and A. Ritieni. 2018. Simultaneous determination of AFB1 and AFM1 in milk samples by ultra high performance liquid chromatography coupled to quadrupole orbitrap mass spectrometry. Beverages 4:43. https://doi.org/10.3390/beverages4020043.

Romera, D., E. M. Mateo, R. Mateo-Castro, J. V. Gómez, J. V. Gimeno-Adelantado, and M. Jiménez. 2018. Determination of multiple mycotoxins in feedstuffs by combined use of UPLC-MS/MS and UPLC-QTOF-MS. Food Chem. 267:140-148. https://doi.org/10 .1016/j.foodchem.2017.11.040.

Rossi, R., G. Saluti, S. Moretti, I. Diamanti, D. Giusepponi, and R. Galarini. 2018. Multiclass methods for the analysis of antibiotic residues in milk by liquid chromatography coupled to mass spectrometry: A review. Food Addit. Contam. A Chem. Anal. Control Expo. Risk Assess. 35:241-257. https://doi.org/10.1080/19440049 .2017.1393107.

Samsidar, A., S. Siddiquee, and S. M. Shaarani. 2018. A review of extraction, analytical and advanced methods for determination of pesticides in environment and foodstuffs. Trends Food Sci. Technol. 71:188-201. https://doi.org/10.1016/j.tifs.2017.11.011.

Serraino, A., F. Giacometti, G. Marchetti, A. V. Zambrini, G. Zanirato, M. Fustini, and R. Rosmini. 2013. Survey on antimicrobial residues in raw milk and antimicrobial use in dairy farms in the Emilia-Romagna region, Italy. Ital. J. Anim. Sci. 12:e68. https:// doi.org/10.4081/ijas.2013.e68.

Singer, R. S., P. L. Ruegg, and D. E. Bauman. 2017. Quantitative risk assessment of antimicrobial-resistant foodborne infections in humans due to recombinant bovine somatotropin usage in dairy cows. J. Food Prot. 80:1099-1116. https://doi.org/10.4315/0362 -028X.JFP-16-404.

Souza-Silva, É. A., E. Gionfriddo, and J. Pawliszyn. 2015. A critical review of the state of the art of solid-phase microextraction of complex matrices II. Food analysis. Trends Anal. Chem. 71:236248. https://doi.org/10.1016/j.trac.2015.04.018.

Speksnijder, D. C., and J. A. Wagenaar. 2018. Reducing antimicrobial use in farm animals: How to support behavioral change of veterinarians and farmers. Anim. Front. 8:4-9. https://doi.org/10.1093/ af/vfy006.

Stolker, A. A. M., and U. T. Brinkman. 2005. Analytical strategies for residue analysis of veterinary drugs and growth-promoting agents in food-producing animals - a review. J. Chromatogr. A 1067:1553. https://doi.org/10.1016/j.chroma.2005.02.037.

Tozzi, B., G. B. Liponi, V. Meucci, L. Casini, C. Dall'Asta, L. Intorre, and D. Gatta. 2016. Aflatoxins M1 and M2 in the milk of donkeys fed with naturally contaminated diet. Dairy Sci. Technol. 96:513523. https://doi.org/10.1007/s13594-016-0285-2.

Tsiplakou, E., C. Anagnostopoulos, K. Liapis, S. A. Haroutounian, and G. Zervas. 2014. Determination of mycotoxins in feedstuffs and ruminant's milk using an easy and simple LC-MS/MS multiresidue method. Talanta 130:8-19. https://doi.org/10.1016/j talanta.2014.06.018.

WHO (World Health Organization). 2017. Stop using antibiotics in healthy animals to prevent the spread of antibiotic resistance. Accessed Oct. 23, 2019. https://www.who.int/news-room/detail/07 -11-2017-stop-using-antibiotics-in-healthy-animals-to-prevent-the -spread-of-antibiotic-resistance.

Xie, J., T. Peng, A. Zhu, J. He, Q. Chang, X. Hu, H. Chen, C. Fan, W. Jiang, M. Chen, J. Li, S. Ding, and H. Jiang. 2015. Multiresidue analysis of veterinary drugs, pesticides and mycotoxins in dairy products by liquid chromatography-tandem mass spectrometry using low-temperature cleanup and solid phase extraction. J. Chromatogr. B Analyt. Technol. Biomed. Life Sci. 1002:19-29. https://doi.org/10.1016/j.jchromb.2015.08.005.

Zhan, J., X. J. Yu, Y. Y. Zhong, Z. T. Zhang, X. M. Cui, J. F. Peng, R. Feng, X. T. Liu, and Y. Zhu. 2012. Generic and rapid determination of veterinary drug residues and other contaminants in raw milk by ultra performance liquid chromatography-tandem mass spectrometry. J. Chromatogr. B Analyt. Technol. Biomed. Life Sci. 906:48-57.

Zhang, Y., X. Li, X. Liu, J. Zhang, Y. Cao, Z. Shi, and H. Sun. 2015. Multi-class, multi-residue analysis of trace veterinary drugs in milk by rapid screening and quantification using ultra-performance liquid chromatography-quadrupole time-of-flight mass spectrometry. J. Dairy Sci. 98:8433-8444. https://doi.org/10.3168/jds.2015-9826.

Zhou, J., J. J. Xu, J. M. Cong, Z. X. Cai, J. S. Zhang, J. L. Wang, and Y.-P. Ren. 2018. Optimization for quick, easy, cheap, effective, rugged and safe extraction of mycotoxins and veterinary drugs by response surface methodology for application to egg and milk. J. Chromatogr. A 1532:20-29. https://doi.org/10.1016/j.chroma .2017.11.050.

Zhu, W. X., J. Z. Yang, Z. X. Wang, C. J. Wang, Y. F. Liu, and L. Zhang. 2016. Rapid determination of 88 veterinary drug residues in milk using automated TurborFlow online clean-up mode coupled to liquid chromatography-tandem mass spectrometry. Talanta 148:401-411. https://doi.org/10.1016/j.talanta.2015.10.037.

\section{ORCIDS}

Luana Izzo @ https://orcid.org/0000-0002-8365-9032

Yelko Rodríguez-Carrasco @ https://orcid.org/0000-0002-6421-218X Josefa Tolosa () https://orcid.org/0000-0003-2260-5257 